\title{
Deep learning prediction of likelihood of ICU admission and mortality in COVID-19 patients using clinical variables
}

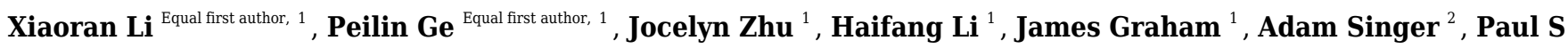 \\ Richman ${ }^{3}$, Tim Q Duong ${ }^{\text {Corresp. } 4}$ \\ ${ }^{1}$ Department of Radiology, Renaissance School of Medicine, Stony Brook University, New York, Stony Brook, New York, United States \\ 2 Department of Emergency Medicine, Renaissance School of Medicine, Stony Brook University, New York, Stony Brook, New York, United States \\ 3 Department of Medicine, Renaissance School of Medicine, Stony Brook University, New York, Stony Brook, New York, United States \\ 4 Department of Radiology, Albert Einstein College of Medicine, Bronx, New York, United States \\ Corresponding Author: Tim Q Duong \\ Email address: tim.duong@einsteinmed.org
}

Background: This study aimed to develop a deep-learning model and a risk-score system using clinical variables to predict intensive care unit (ICU) admission and in-hospital mortality in COVID-19 patients. Methods: This retrospective study consisted of 5766 persons-under-investigation for COVID-19 between February 7, 2020, and May 4, 2020. Demographics, chronic comorbidities, vital signs, symptoms, and laboratory tests at admission were collected. A deep neural network model and a risk-score system were constructed to predict ICU admission and in-hospital mortality. Prediction performance used the receiver operating characteristic area under the curve (AUC). Results: The top ICU predictors were procalcitonin, lactate dehydrogenase, C-reactive protein, ferritin, and oxygen saturation. The top mortality predictors were age, lactate dehydrogenase, procalcitonin, cardiac troponin, C-reactive protein, and oxygen saturation. Age and troponin were unique top predictors for mortality but not ICU admission. The deep-learning model predicted ICU admission and mortality with an AUC of 0.780 [95\% Cl:0.760-0.785] and 0.844 [95\% Cl:0.839-0.848], respectively. The corresponding risk scores yielded an AUC of 0.728 [95\% Cl:0.726-0.729] and 0.848 [95\% Cl:0.847-0.849],

respectively.Conclusions: Deep learning and the resultant risk score have the potential to provide frontline physicians with quantitative tools to stratify patients more effectively in time-sensitive and resource-constrained circumstances. 
1

2

3

4

5

6

7

8

9

10

11

12

13

14

15

16

17

20

21

22

23

24

25

26

27

28

29

30

31

32

33

34

35

36

37

38

39

40

41

42

Manuscript Type: Original research

\section{Deep learning prediction of likelihood of ICU admission and mortality in COVID-19 patients using clinical variables}

Xiaoran $\mathrm{Li}^{1 *}$, Peilin $\mathrm{Ge}^{1 *}$, Jocelyn $\mathrm{Zhu}^{1 *}$, Haifang $\mathrm{Li}^{1}$, James Graham, Adam J. Singer ${ }^{2}$, Paul S. Richman ${ }^{3}$, Tim Q Duong ${ }^{4}$

${ }^{*}$ Co-first authors

${ }^{1}$ Department of Radiology, Renaissance School of Medicine, Stony Brook University, New York

${ }^{2}$ Department of Emergency Medicine, Renaissance School of Medicine, Stony Brook University, New York

${ }^{3}$ Department of Medicine, Renaissance School of Medicine, Stony Brook University, New York

${ }^{4}$ Department of Radiology, Albert Einstein College of Medicine, Bronx, New York

Correspondence: Tim Duong, PhD, Radiology, ${ }^{4}$ Department of Radiology, Albert Einstein College of Medicine, 111 E 210th St, Bronx, New York, Tim.duong@einsteinmed.org, T 7189206268

Word Count for Text: 2800

Key words: Machine learning, coronavirus, SARS-CoV-2, prediction model.

\section{ACKNOWLEDGEMENT}

We thank all healthcare professionals for their hard work being at the front line of the pandemic. All authors had full access to data, and declared no conflict of interest, including financial interests, activities, relationships, and affiliations. We report no sources of funding.

\section{Funding None}

Disclaimer NA

Competing interests none declared.

Ethics approval. Our institutional review board (Stony Brook University) approved this retrospective study, and the requirement for informed consent was waived.

Abbreviations: alanine aminotransferase (ALT), C-reactive protein (CRP), lactate dehydrogenase (LDH), white blood cells (WBC), real-time polymerase chain reaction (RT-PCR), area under the curve (AUC) 


\section{ABSTRACT}

44 Background: This study aimed to develop a deep-learning model and a risk-score system using clinical 45 variables to predict intensive care unit (ICU) admission and in-hospital mortality in COVID-19 patients.

46 Methods: This retrospective study consisted of 5766 persons-under-investigation for COVID-19 between

47 February 7, 2020, and May 4, 2020. Demographics, chronic comorbidities, vital signs, symptoms, and

48 laboratory tests at admission were collected. A deep neural network model and a risk-score system were

49 constructed to predict ICU admission and in-hospital mortality. Prediction performance used the receiver 50 operating characteristic area under the curve (AUC).

51 Results: The top ICU predictors were procalcitonin, lactate dehydrogenase, C-reactive protein, ferritin, 52 and oxygen saturation. The top mortality predictors were age, lactate dehydrogenase, procalcitonin, 53 cardiac troponin, C-reactive protein, and oxygen saturation. Age and troponin were unique top predictors 54 for mortality but not ICU admission. The deep-learning model predicted ICU admission and mortality 55 with an AUC of 0.780 [95\% CI:0.760-0.785] and 0.844 [95\% CI:0.839-0.848], respectively. The 56 corresponding risk scores yielded an AUC of 0.728 [95\% CI:0.726-0.729] and 0.848 [95\% CI:0.84757 0.849], respectively.

58 Conclusions: Deep learning and the resultant risk score have the potential to provide frontline physicians 59 with quantitative tools to stratify patients more effectively in time-sensitive and resource-constrained 60 circumstances. 
62

63

64

65

66

67

68

69

70

71

72

73

74

75

76

77

78

79

80

81

82

83

84

85

86

87

88

89

90

91

92

93

94 95

\section{INTRODUCTION}

Since the first reports of severe respiratory illness caused by coronavirus disease 2019 (COVID19) in Wuhan, China in mid-December 2019 (Huang et al. 2020; Zhu et al. 2020), over 6.2 million individuals have been infected, resulting in over 370,000 deaths worldwide (May 31, 2020). The actual numbers are likely to be much higher due to testing shortages and under-reporting (Yelin et al. 2020). Many patients have mild or asymptomatic infections, while others deteriorate rapidly with multi-organ failure. There will likely be recurrence and secondary waves of this pandemic (Leung et al. 2020).

A large array of clinical and demographic variables associated with COVID-19 infection have been identified (see reviews (Brown et al. 2020; Cao et al. 2020; Rodriguez-Morales et al. 2020)). A few of these have been associated with high likelihood of critical illness or mortality. There are however no established prognostic models that reliably predict the need for escalated (intensive care unit, ICU) care or mortality due to COVID-19 infection. Lacking this, effective triage of patients is challenging in a resource-constrained environment. The problem is further magnified by the poor sensitivity (Kim et al. 2020 , in press) and a few day turnaround time (Yelin I 2020) of the most commonly used reversetranscriptase polymerase chain reaction (RT-PCR) test, during which time patients are assumed COVID19 positive. This problem strains the resources of many hospitals and highlights the need for effective tools to anticipate patients' progression and properly triage patients.

The goal of this study was to develop a deep-learning algorithm (in contrast to previous methods) to identify the top, statistically significant predictors amongst the large array of clinical variables at admission to predict the likelihood of ICU admission and in-hospital mortality in COVID-19 patients. We further developed a simplified risk-score model to predict the likelihood of ICU admission and in-hospital mortality.

\section{METHODS}

Study population

This retrospective study was approved by Institutional Review Board with exemption of informed consent and HIPAA waiver (Stony Brook University Hospital, IRB-2020-00207). Stony Brook University Hospital, the only academic hospital serving Suffolk county, about 40 miles east of New York City, was one of the hardest hit counties in the country at the time of this writing. The COVID-19 Persons Under Investigation (PUI) registry consisted of 5766 patients from February 7th, 2020 to May 4th, 2020. Only patients who were diagnosed by positive tests of real-time polymerase chain reaction (RT-PCR) for severe acute respiratory syndrome coronavirus 2 (SARS-CoV-2) were included in the study.

94 Demographic information, chronic comorbidities, imaging findings, vital signs, symptoms, and laboratory tests at admission were collected. Imaging findings were extracted from patient chart review, which 
96 included information provided by radiology report as part of standard of care. The primary outcome was

97 ICU admission versus general floor admission, and the secondary outcome was in-hospital mortality

98 versus discharge. Mortality outside of hospital after discharge was not obtained.

99 Figure 1 shows the flowchart of patient selection. Of the 2594 confirmed COVID-19 positive

100 cases, all 1108 hospitalized COVID-19 positive patients were used in our analysis. Seventy-seven (77)

101 patients were admitted to the ICU directly and an additional 194 patients were subsequently upgraded to

102 an ICU from a general floor. Among these 271 ICU patients, 108 were discharged alive, 77 expired

103 during the hospitalization and the other 86 are still in the hospital at the time of this analysis. Comparison

104 was made to 837 general admissions who did not receive ICU care, among whom 772 patients were

105 discharged alive and 65 expired during the hospitalization (none remained in the hospital).

106

107 Data preprocessing

108 Two patients were excluded from machine learning analysis for missing categorical variables.

109 Brain natriuretic peptide (BNP) was missing from $>15 \%$ of patients, thus they were excluded from

110 machine learning analysis. For the rest of the laboratory variables, missing data (in $<5 \%$ of patients) was

111 imputed with predictive mean modeling using the Multivariate Imputation by Chained Equations in $\mathrm{R}$

112 (statistical analysis software, version 4.0) (van Buuren \& Groothuis-Oudshoorn 2011).

113

114 Deep neural network prediction model

115 Ranking of clinical variables of categorical or numerical values were made using the Boruta, a

116 statistical software (Kursa \& Rudnicki 2010). Boruta ranks feature importance using the Random Forest

117 method. In this decision tree-based method, the quantitative measure of importance is the Gini feature of

118 importance, which counts the times that a feature is used to split a node of a decision tree, statistically

119 weighted by the number of instances the node splits. In the DNN model, the top predictors were those that

120 demonstrated statistical significance using built-in statistical methods within the Boruta algorithm.

121 A correlation coefficient $>0.5$ from collinearity analysis was used to exclude correlated variables

122 from machine learning analysis. Note that none of the top features we used in the final analysis

123 demonstrated strong correlation with other features. Thus, no top features were removed as a result. A

124 deep neural network (DNN) was constructed to predict ICU admission and mortality using five fully

125 connected dense layers (Chen et al. 2020). The top clinical predictors were input parameters, determined

126 by testing subsets of these parameters, and ICU admission and mortality were outcome parameters. The

127 DNN model used 5 hidden layers with $6,8,16,8,4$ neurons respectively. We explored a few models

128 using a range of number (3-7) of layers, and the 5-layer model yielded the optimal validation result. ReLu

129 activation function for the hidden layers, the sigmoid activation function for the output layer, and the

Peer] reviewing PDF | (2020:07:51182:1:1:CHECK 18 Sep 2020) 
130 "he_normal" normalization scheme were applied. In the model training process, we used Adam optimizer,

131 mean squared error as the cost function, a default learning rate of 0.01 , and number of epochs of 100 . The

132 reported results yielded from the average of 5 consecutive runs. The dataset was randomly split into $90 \%$

133 training data and 10\% testing data. ICU admission and mortality results were categorized using a binary

134 classification. To minimize overfitting, we employed 5-fold cross-validation, ranked and removed less

135 important features using correlation analysis and based on statistical significance by Boruta. We also

136 employed regularization and stopped the training process at 100 epochs.

137

138 Risk score model

139 Risk-score systems were constructed using the top independent clinical variables to predict ICU

140 admission and mortality. For risk score, the mixed Generalized Additive Model was used to plot the

141 probability of ICU admission and mortality for each clinical variable (Wood 2001). Different cutoff

142 points were evaluated where the chosen cutoff points yielded the optimal distribution (not skewed to high

143 or low scores) of the risk score model. The corresponding numerical values of each top feature at

144 probability of 0.3 for ICU and 0.2 for mortality were found to be the optimal cutoff values for the risk

145 score model. Each of the top variables was assigned a weight of one point if the clinical measurement was

146 above the probability cutoff. The risk score ranged from 0 to 5 for ICU admission and 0 to 6 for mortality

147 (which were chosen based on statistical significance, see Results).

148

149 Statistical analysis and performance evaluation

150 Statistical analysis was performed in SPSS v26 and in R (statistical analysis software 4.0). Group

151 comparisons of categorical variables in frequencies and percentages used the chi-square test or Fisher

152 exact test. Group comparison of continuous variables in medians and interquartile ranges (IQR) used the

153 Mann-Whitney $U$ test. A p value $<0.05$ was considered to be statistically significant. For performance

154 evaluation, data were split $90 \%$ for training and $10 \%$ for testing. Prediction performance was evaluated by

155 calculating the area under the curve (AUC) of the receiver operating characteristic (ROC) curve,

156 accuracy, sensitivity, specificity, precision, recall, negative predictive value (NPV), positive predictive

157 value (PPV) and F1 score (a harmonic mean of precision and recall). The average ROC analysis was

158 repeated with five runs. In risk score models, SPSS was used to cross-check statistical significance of the

159 top features, in which all top features used in the final analysis of risk score model had a $\mathrm{p}<0.001$.

160

161

162 RESULTS

163

Peer] reviewing PDF | (2020:07:51182:1:1:CHECK 18 Sep 2020) 
164

165

166

167

168

169

170

171

172

173

174

175

176

177

178

179

180

181

182

183

184

185

186

187

188

189

190

191

192

193

194

195

196

197

\section{Clinical variables associated with ICU admission}

Table 1 summarizes the demographic characteristics, vital signs, comorbidities, and laboratory data for the ICU $(n=271)$ and non-ICU $(n=837)$ group. The median age of the ICU group was lower than that of the general admission group (59 years [IQR:49-71] versus 62 years [IQR:50-76], $\mathrm{p}=0.027$ ). Disproportionally more males were admitted to the ICU $(67.5 \% \%$ vs $32.5 \%, \mathrm{p}<0.001)$. History of cancer was the only comorbidity that was significantly associated with ICU admission ( $\mathrm{P}=0.016)$.

All measured vital signs were significantly different between the ICU group and the non-ICU group. The ICU group had higher heart rate, respiratory rate and temperature, but lower systolic blood pressure and oxygen saturation $(\mathrm{p}<0.05)$. The ICU group had higher alanine aminotransferase (ALT), Creactive protein (CRP), D-dimer, ferritin, lactate dehydrogenase (LDH), white blood cells (WBC), and procalcitonin $(\mathrm{p}<0.05)$ and lower lymphocyte counts $(\mathrm{p}<0.05)$. Cardiac troponin and BNP were not significantly different between groups $(\mathrm{p}>0.05)$.

The symptom of dyspnea was significantly associated with ICU admission $(p=0.001)$. Patients admitted to ICU were more likely to present with abnormal chest $\mathrm{x}$-ray $(\mathrm{p}<0.001)$, and more likely to have bilateral chest $\mathrm{x}$-ray abnormalities on presentation, compared to that of general admission group $(\mathrm{p}<0.001)$.

\section{Prediction models for ICU admission}

Figure 2 shows the ranking of the clinical variables associated with ICU admission. The top 5 statistically significant predictors of ICU admission were procalcitonin, LDH, CRP, ferritin, and SpO2. A deep neural network predictive model for mortality was constructed using the top clinical variables and trained using the training dataset and tested on an independent testing dataset. The ROC and confusion matrix of the testing dataset are shown in Figure 3. The performance of the DNN model yielded an AUC $=0.780$ [95\% CI:0.760-0.785], sensitivity $=0.760$, specificity $=0.709$, and $\mathrm{F} 1$ score $=0.551$ in predicting ICU admission for the testing set (Table 2).

A risk score system was constructed (training data set) using the top five statistically significant clinical variables, with 1 point given for each variable meeting the following criteria: procalcitonin $>0.5 \mathrm{ng} / \mathrm{mL}, \mathrm{LDH}>487 \mathrm{U} / \mathrm{L}$ and $<12586.7 \mathrm{U} / \mathrm{L}, \mathrm{CRP}>14.2 \mathrm{mg} / \mathrm{dL}$, ferritin $>1250 \mathrm{ng} / \mathrm{mL}$ and $<13080.5 \mathrm{ng} / \mathrm{mL}$, and $\mathrm{SpO} 2<88.8 \%$. Odds ratios of procalcitonin, $\mathrm{LDH}, \mathrm{CRP}$, ferritin, and $\mathrm{SpO} 2$ for ICU admission were 3.062, 3.846, 3.001, 2.449, and 3.665, respectively. Figure 4 shows the results for the testing data set using the risk score system. ICU admission rate increased with increasing risk scores. The performance of the risk score yielded an AUC of 0.728 [95\% CI:0.726-0.729] for predicting ICU admission for the testing data set. 
198

199

200

201

202

203

204

205

206

207

208

209

210

211

212

213

214

215

216

217

218

219

220

221

222

223

224

225

226

227

228

229

230

231

Clinical variables associated with mortality

Table 3 summarizes the demographic data, vital signs, comorbidities, and laboratory data for the non-survivors $(n=142)$ and survivors $(n=880)$ group. The median age of the non-survivor group was higher than that of the survivor group (76 years [IQR:66-84] versus 59 years [IQR:49-72], $\mathrm{p}<0.001$ ). There was a disproportionally higher mortality rate in males $(65.5 \%$ vs $34.5 \%, p=0.014)$. Of the comorbidities, hypertension, coronary artery disease, heart failure, chronic obstructive pulmonary disease, smoking history, and chronic kidney disease were significantly different between groups $(\mathrm{p}<0.05)$.

Among vital signs, tachypnea and hypoxemia were significantly different between groups at presentation $(\mathrm{p}<0.05)$. The expired cohort had higher BNP, CRP, D-dimer, ferritin, LDH, WBC, procalcitonin, and cardiac troponin but lower lymphocytes $(\mathrm{p}<0.05)$. ALT was not significantly different between groups.

Among the symptoms, cough, myalgia, nausea or vomiting, chest discomfort, fatigue, fever, loss of taste, and headache were significantly different between groups $(\mathrm{p}<0.05)$. There was no significant difference in $\mathrm{x}$-ray findings between groups at presentation.

\section{Prediction models for mortality}

The top 6 statistically significant predictors of mortality were age, $\mathrm{LDH}$, procalcitonin, troponin, $\mathrm{CRP}$, and SpO2 (Figure 5). A deep neural network predictive model for mortality was constructed using the top clinical variables and trained using the training data set. The ROC and confusion matrix are shown in Figure 6. The performance of the DNN model yielded an AUC of 0.844 [95\% CI:0.839-0.848], sensitivity $=0.750$, specificity $=0.872$, and F1 score $=0.616$ for the testing dataset (Table 4).

A risk score system was constructed (training data set) using the top 6 statistically significant clinical variables to predict mortality. The thresholds for the risk scores were: age $>71$ years, LDH $>487 \mathrm{U} / \mathrm{L}$, procalcitonin $>1.1 \mathrm{ng} / \mathrm{mL}$, troponin $>0.03 \mathrm{ng} / \mathrm{mL}, \mathrm{CRP}>17 \mathrm{mg} / \mathrm{dL}$, and $\mathrm{SpO} 2<88 \%$. Odds ratios of age, $\mathrm{LDH}$, procalcitonin, troponin, CRP, and $\mathrm{SpO} 2$ for mortality were 4.301, 3.418, 6.232, 5.253, 4.240, and 3.750, respectively. Higher mortality rate was associated with higher risk scores for the testing set (Figure 7). The performance of the risk score yielded an AUC of 0.848 [95\% CI:0.847-0.849] in predicting mortality for the testing set.

\section{DISCUSSION}

Mining a large cohort of COVID-19 patients in the United States, deep-learning and resultant risk score models identified the top predictors of ICU admission in COVID-19 to be the admission levels of procalcitonin, $\mathrm{LDH}, \mathrm{CRP}$, ferritin, and $\mathrm{SpO} 2$; the top predictors of mortality were age, LDH, procalcitonin, cardiac troponin, CRP, and SpO2. Predictive models were developed using deep neural 
232

233

234

235

236

237

238

239

240

241

242

243

244

245

246

247

248

249

250

251

252

253

254

255

256

257

258

259

260

261

262

263

264

265

network of the top predictors, yielding an AUC of 0.779 and 0.882 for predicting ICU admission and mortality, respectively. The corresponding simplified risk scores yielded an AUC of 0.728 and 0.848 , respectively.

The association between these biomarkers and poor outcomes in COVID-19 victims is biologically plausible: procalcitonin is elevated during bacterial infection, but less so during viral infection, suggesting that bacterial co-infection leads to worse outcome in COVID-19 patients (Assicot et al. 1993). LDH reflects tissue damage (Huang et al. 2020; Zhu et al. 2020), while CRP is indicative of inflammation (Gabay \& Kushner 1999). Elevated ferritin is associated with acute respiratory distress syndrome (ARDS) (Connelly et al. 1997) and may be a marker of aberrant iron metabolism that could render the lungs susceptible to oxidative damage (Mumby et al. 2004). Ferritin may reflect hyperinflammation associated with a cytokine storm and multi-organ failure (Mehta et al. 2020). Low $\mathrm{SpO} 2$ indicates failure of the lungs to oxygenate blood effectively, leading to tissue hypoxia (Connelly et al. 1997). Elevated cardiac troponin indicates cardiac injury (Huang et al. 2020). Although these variables have been previously associated with COVID-19 infection, most previous studies did not rank these clinical variables, or develop predictive models or risk scores to predict ICU admission or mortality. Not surprisingly, some of the same biomarkers in our study predicted both the need for ICU admission and likelihood of mortality. However, age and admission troponin level were uniquely predictive of mortality, indicating older age and cardiac issues are associated with higher rate of mortality in COVID-19 infection.

It is notable that individual comorbidities did not rank high in predicting ICU admission and mortality. Specifically, a history of heart failure, COPD, and coronary artery disease only ranked $7^{\text {th }}, 11^{\text {th }}$ and $14^{\text {th }}$ respectively for predicting mortality. Similarly, the patients' symptoms and vital signs (other than $\mathrm{SpO} 2$ ) at the time of admission were not found to be the top predictors of poor outcome. Although some comorbidities have been reported to be associated with critical illness and mortality, most previously studies did not rank their importance with respect to other laboratory variables.

Our predictive AUC performance for ICU admission was poorer than that for mortality. We speculate this might be due to variability in triage decision-making to send patients to ICU among frontline clinicians. For both predictions, precision, PPV and F1 scores were comparatively low, which was not unexpected due to the imbalanced sample sizes between the two groups as well as small sample sizes. Further studies are warranted.

While a large number of studies have previously identified clinical variables associated with the severity of COVID-19 infection, only a few studies have attempted to develop a predictive or risk score model to predict mortality and disease severity. Jiang et al. used supervised learning (not deep learning) 
266

267

268

269

270

271

272

273

274

275

276

277

278

279

280

281

282

283

284

285

286

287

288

289

290

291

292

293

294

295

296

297

298

299

and found mildly elevated alanine aminotransferase, myalgias, and hemoglobin at presentation to be predictive of severe ARDS of COVID-19 with $70 \%$ to $80 \%$ accuracy. This study had small, non-uniform, heterogeneous clinical variables, obtained from different hospitals (Jiang et al. 2020). Ji et al. used logistic regression to predict stable versus progressive COVID-19 patients $(n=208)$ based on whether their conditions worsened during hospitalization (Ji et al. 2020). They reported comorbidities, older age, lower lymphocyte and higher lactate dehydrogenase at presentation to be independent high-risk factors for COVID-19 progression but did not develop a risk score. A nomogram of these 4 factors yielded a concordance index of 0.86. Yan et al. utilized supervised machine learning to predict critical COVID-19 at admission using presence of X-ray abnormality, cancer history, age, neutrophil/lymphocyte ratio, LDH, dyspnea, bilirubin, unconsciousness and number of comorbidities (Yan et al. 2020, in press). They reported an AUC of 0.88. Yuan et al. went one step further to predict mortality more than 12 days in advance with $>90 \%$ accuracy across all cohorts. Moreover, their Kaplan-Meier score shows that patients upon admission could clearly be differentiated into low, medium or high risk. They created a simple risk score system, and validated using multiple independent cohorts (Yuan et al. 2020).

Our approach used a deep-learning algorithm which is novel and has distinct advantages over logistic regression and supervised learning approach. Deep learning is increasingly being used in medicine (Deo 2015; Santos et al. 2019; Tschandl et al. 2019). In contrast to conventional analysis methods, which specify the relationships amongst data elements to outcomes, machine learning employs computer algorithms to identify relationships amongst different data elements to inform outcomes without the need to specify such relationships a priori. Deep learning can outperform human experts in performing many tasks in medicine (Killock 2020). In addition to approximating physician skills, Deep learning can also detect novel relationships not readily apparent to human perception, especially in large, complex, and longitudinal datasets. Disadvantages of deep learning methods are that it requires comparatively large sample size, there is a potential of overfitting, and the complex relations could make deep learning results difficult to interpret, amongst others. In addition, we devised a simplified practical risk score adds practical utility to these findings. Although we ranked all variables and explicitly listed 10 or 15 top variables, we built the predictive model and risk score model using only the top 5 variables to simplify and increase translation potential in the clinical settings. The excellent prediction performances using a few clinical variables are encouraging.

This study has several limitations in addition to those mentioned above. This is a retrospective study carried out in a single hospital. These findings need to be replicated in large and multi-institutional settings for generalizability. We only analyzed clinical variables at admission. Longitudinal changes of these clinical variables need to be studied. As in all observational studies, other residual confounders may 
300 exist that were not accounted for in our analysis. Future prospective studies validating our predictive

301 models and scores are warranted.

302

303 CONCLUSION

304 We implemented a deep-learning algorithm and a risk score model to predict the likelihood of ICU 305 admission and mortality in COVID-19 patients. Our predictive model and risk score model can be easily 306 retrained with additional data, new local data, as well as additional clinical variables. This approach has

307 the potential to provide frontline physicians with a simple and objective tool to stratify patients based on 308 risks so that COVID-19 patients can be triaged more effectively in time-sensitive, stressful and potentially 309 resource-constrained environments.

310

\section{ACKNOWLEDGEMENT}

312

The work has been approved by Stony Brook Institutional Review Board. We thank all healthcare

313 Professionals for their hard work being at the front line of the pandemic. All authors had full access to

314 data, and declared no conflict of interest, including financial interests, activities, relationships, and

315 affiliations. We report no sources of funding.

316

317

318

319

320 


\section{REFERENCES}

322 https://coronavirus.jhu.edu/map.html. assessed Jul 11, 2020.

323

324

325

326

327

Assicot M, Gendrel D, Carsin H, Raymond J, Guilbaud J, and Bohuon C. 1993. High serum procalcitonin concentrations in patients with sepsis and infection. Lancet 341:515-518. 10.1016/0140-6736(93)90277-n

Brown RAC, Barnard J, Harris-Skillman E, Harbinson B, Dunne B, Drake J, Roche S, Harris E, Gunnel J, Frost J, Angus B, and Hodgson S. 2020. Lymphocytopaenia is associated with severe SARS-CoV-2 disease: A Systematic Review and Meta-Analysis of Clinical Data. medRxiv:2020.2004.2014.20064659. 10.1101/2020.04.14.20064659

Cao Y, Liu X, Xiong L, and Cai K. 2020. Imaging and Clinical Features of Patients With 2019 Novel Coronavirus SARS-CoV-2: A systematic review and meta-analysis. Journal of Medical Virology 03:03. https://dx.doi.org/10.1002/jmv.25822

Chen Z, Pang M, Zhao Z, Li S, Miao R, Zhang Y, Feng X, Feng X, Zhang Y, Duan M, Huang L, and Zhou F. 2020. Feature selection may improve deep neural networks for the bioinformatics problems. Bioinformatics 36:1542-1552. 10.1093/bioinformatics/btz763

Connelly KG, Moss M, Parsons PE, Moore EE, Moore FA, Giclas PC, Seligman PA, and Repine JE. 1997. Serum ferritin as a predictor of the acute respiratory distress syndrome. American Journal of Respiratory \& Critical Care Medicine 155:21-25. 10.1164/ajrccm.155.1.9001283

Deo RC. 2015. Machine Learning in Medicine. Circulation 132:1920-1930. 10.1161/CIRCULATIONAHA.115.001593

Gabay C, and Kushner I. 1999. Acute-phase proteins and other systemic responses to inflammation. New England Journal of Medicine 340:448-454. 10.1056/NEJM199902113400607

Huang C, Wang Y, Li X, Ren L, Zhao J, Hu Y, Zhang L, Fan G, Xu J, Gu X, Cheng Z, Yu T, Xia J, Wei Y, Wu W, Xie X, Yin W, Li H, Liu M, Xiao Y, Gao H, Guo L, Xie J, Wang G, Jiang R, Gao Z, Jin Q, Wang J, and Cao B. 2020. Clinical features of patients infected with 2019 novel coronavirus in Wuhan, China. Lancet 395:497-506. 10.1016/S0140-6736(20)30183-5

Ji D, Zhang D, Xu J, Chen Z, Yang T, Zhao P, Chen G, Cheng G, Wang Y, Bi J, Tan L, Lau G, and Qin E. 2020. Prediction for Progression Risk in Patients with COVID-19 Pneumonia: the CALL Score. Clinical Infectious Diseases 09:09. https://dx.doi.org/10.1093/cid/ciaa414

Jiang X, Coffee2 M, Bari A, Wang J, Jiang X, Huang J, Shi J, Dai J, Cai J, Zhang, T., Wu Z, He G, and Huang Y. 2020. Towards an Artificial Intelligence Framework for Data-Driven Prediction of Coronavirus Clinical Severity. Computers, Materials \& Continua 63:537-551.

Killock D. 2020. AI outperforms radiologists in mammographic screening. Nat Rev Clin Oncol 17:134. 10.1038/s41571-020-0329-7

Kim HA, Hong HE, and Yoon SH. 2020, in press. Diagnostic performance of CT and RT-PCR for COVID-19: A meta-analysis. Radiology.

Kursa MB, and Rudnicki WR. 2010. Feature Selection with the Boruta Package. Journal of Statistical Software 36:1-13.

Leung K, Wu JT, Liu D, and Leung GM. 2020. First-wave COVID-19 transmissibility and severity in China outside Hubei after control measures, and second-wave scenario planning: a modelling impact assessment. The Lancet 395:1382-1393. 10.1016/S0140-6736(20)30746-7

Mehta P, McAuley DF, Brown M, Sanchez E, Tattersall RS, Manson JJ, and Hlh Across Speciality Collaboration UK. 2020. COVID-19: consider cytokine storm syndromes and immunosuppression. Lancet 395:10331034. 10.1016/S0140-6736(20)30628-0

Mumby S, Upton RL, Chen Y, Stanford SJ, Quinlan GJ, Nicholson AG, Gutteridge JM, Lamb NJ, and Evans TW. 2004. Lung heme oxygenase-1 is elevated in acute respiratory distress syndrome. Crit Care Med 32:11301135. 10.1097/01.ccm.0000124869.86399.f2

Rodriguez-Morales AJ, Cardona-Ospina JA, Gutiérrez-Ocampo E, Villamizar-Peña R, Holguin-Rivera Y, EscaleraAntezana JP, Alvarado-Arnez LE, Bonilla-Aldana DK, Franco-Paredes C, and Henao-Martinez AF. 2020. Clinical, laboratory and imaging features of COVID-19: A systematic review and meta-analysis. Travel medicine and infectious disease:101623. 10.1016/j.tmaid.2020.101623

Santos MK, Ferreira Junior JR, Wada DT, Tenorio APM, Barbosa MHN, and Marques PMA. 2019. Artificial intelligence, machine learning, computer-aided diagnosis, and radiomics: advances in imaging towards to precision medicine. Radiol Bras 52:387-396. 10.1590/0100-3984.2019.0049 
374

375

376

377

378

379

380

381

382

383

384

385

386

387

388

389

390

391

392

393

394

395

396

397

398

399
Tschandl P, Codella N, Akay BN, Argenziano G, Braun RP, Cabo H, Gutman D, Halpern A, Helba B, HofmannWellenhof R, Lallas A, Lapins J, Longo C, Malvehy J, Marchetti MA, Marghoob A, Menzies S, Oakley A, Paoli J, Puig S, Rinner C, Rosendahl C, Scope A, Sinz C, Soyer HP, Thomas L, Zalaudek I, and Kittler H. 2019. Comparison of the accuracy of human readers versus machine-learning algorithms for pigmented skin lesion classification: an open, web-based, international, diagnostic study. Lancet Oncol 20:938-947. 10.1016/S1470-2045(19)30333-X

van Buuren S, and Groothuis-Oudshoorn K. 2011. mice: Multivariate Imputation by Chained Equations in R. Journal of Statistical Software 45:1-67.

Wood SN. 2001. mgcv: GAMs and generalized ridge regression for R. 1.

Yan L, Zhang HT, Goncalves J, Xiao Y, Wang M, Guo Y, Sun C, Tang X, Jing L, Zhang M, Huang X, Xiao Y, Cao H, Chen Y, Ren T, Wang F, Xiao Y, Huang S, Tan X, Huang N, Jiao B, Cheng C, Zhang Y, Luo A, Mombaerts L, J. J, and Cao Z. 2020, in press. An interpretable mortality prediction model for COVID-19 patients. Nat Machine Intelligence.

Yelin I, Aharony N, Shaer-Tamar E, Argoetti A, Messer E, Berenbaum D, Shafran E, Kuzli A, Gandali N, and Hashimshony T. 2020. Evaluation of COVID-19 RT-qPCR test in multi-sample pools. medRxiv.

Yelin I AN, Tamer ES, Argoetti, Messer E, Berenbaum, Shafran E, Kuzli A, Gandali, Hashimshony T, MandelGutfreund Y, Halberthal M, Geffen Y, Szwarcwort-Cohen M, Kishony R. 2020. Evaluation of COVID-19 RT-qPCR test in multi-sample pools. https://doi.org/10.1101/2020.03.26.20039438

Yuan Y, Sun C, Tang X, Cheng C, Mombaerts L, Wang M, Hu T, Sun C, Guo Y, Li X, Xu H, Ren T, Xiao Y, Xiao Y, Zhu H, Chen C, Liu Y, Liang Z, Cao Z, Zhang HT, Paschaldis IC, Liu Q, Gonalves J, Zhong Q, and Yan L. 2020. Development and validation of a prognostic risk score system for COVID-19 inpatients: A multicenter retrospective study in China. Pre-print. 10.21203/rs.3.rs-41151/v1

Zhu N, Zhang D, Wang W, Li X, Yang B, Song J, Zhao X, Huang B, Shi W, Lu R, Niu P, Zhan F, Ma X, Wang D, $\mathrm{Xu} \mathrm{W}, \mathrm{Wu}$ G, Gao GF, Tan W, China Novel Coronavirus I, and Research T. 2020. A Novel Coronavirus from Patients with Pneumonia in China, 2019. N Engl J Med 382:727-733. 10.1056/NEJMoa2001017 
400

401

402

403

404

405

406

407

408

409

410

411

412

413

414

415

416

417

418

419

420

421

422

423

424

425

426

427

428

429

430

\section{Figure legends}

Figure 1. Patient selection flowchart.

Figure 2. Ranking of clinical variables for predicting ICU admission by Boruta algorithm. The $\mathrm{x}$-axis is attribute of level of importance, where a larger number indicates relatively higher importance. The y-axis are laboratory test variables. The top statistically significant predictors were: procalcitonin, LDH, CRP, ferritin, $\mathrm{SpO} 2$, lymphocytes, respiratory rate, systolic blood pressure, age and ALT. The top 10 variables were significant.

Figure 3. ROC and confusion matrix for prediction of ICU admission of the DNN model.

Figure 4. Risk score stratification for ICU admission. Scores ranged from 0 to 5 , with 0 indicating the lowest risk and 5 being the highest risk of mortality. The numbers in the bar indicate the number of patients in the ICU (red) and non-ICU (blue) that were correctly predicted in the testing dataset.

Figure 5. Ranking of clinical variables for predicting mortality by Boruta algorithm. The $\mathrm{x}$-axis is attribute of level of importance, where a larger number indicates relatively higher importance. The y-axis are laboratory test variables. The top statistically significant predictors were: age, LDH, procalcitonin, troponin, $\mathrm{CRP}, \mathrm{SpO} 2$, history of heart failure, respiratory rate, lymphocytes, ferritin, history of COPD, Ddimer, ALT, history of coronary heart disease, and systolic blood pressure. The top 15 variables were significant.

Figure 6. ROC and confusion matrix for prediction of mortality of the DNN model.

Figure 7. Risk score stratification for mortality. Scores ranged from 0 to 6 , with 0 indicating the lowest risk and 6 being the highest risk of mortality. The numbers in the bar indicate the number of patients in the ICU (red) and non-ICU (blue) that were correctly predicted in the testing dataset.

Peer] reviewing PDF | (2020:07:51182:1:1:CHECK 18 Sep 2020) 
Figure 1

Patient selection flowchart.

Patient selection flowchart. 


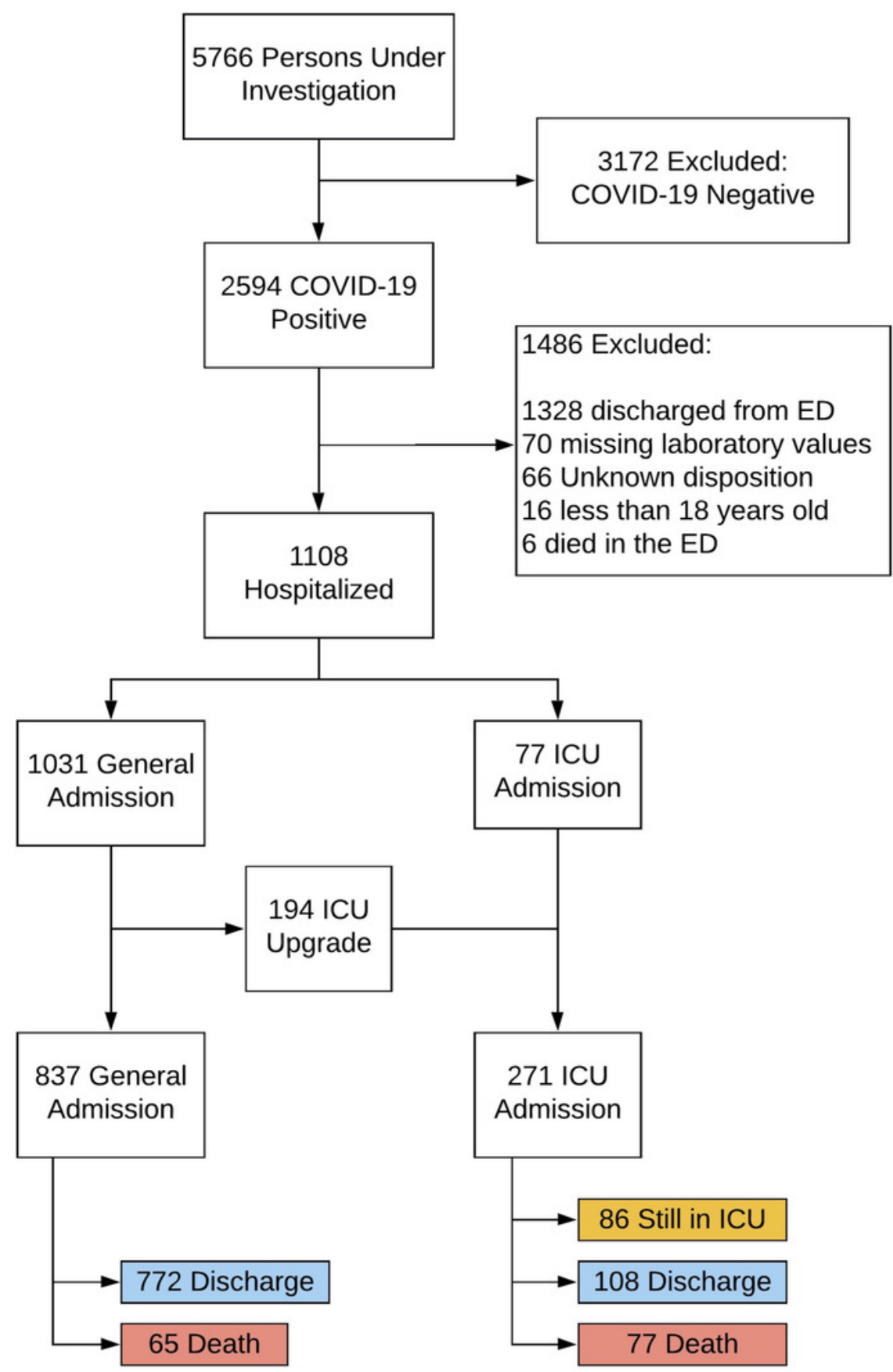




\section{Figure 2}

Ranking of clinical variables for predicting ICU admission

Ranking of clinical variables for predicting ICU admission by Boruta algorithm. The $\mathrm{x}$-axis is attribute of level of importance, where a larger number indicates relatively higher importance. The y-axis are laboratory test variables. The top statistically significant predictors were: procalcitonin, LDH, CRP, ferritin, SpO2, lymphocytes, respiratory rate, systolic blood pressure, age and ALT. The top 10 variables were significant. 


\section{ICU admission}

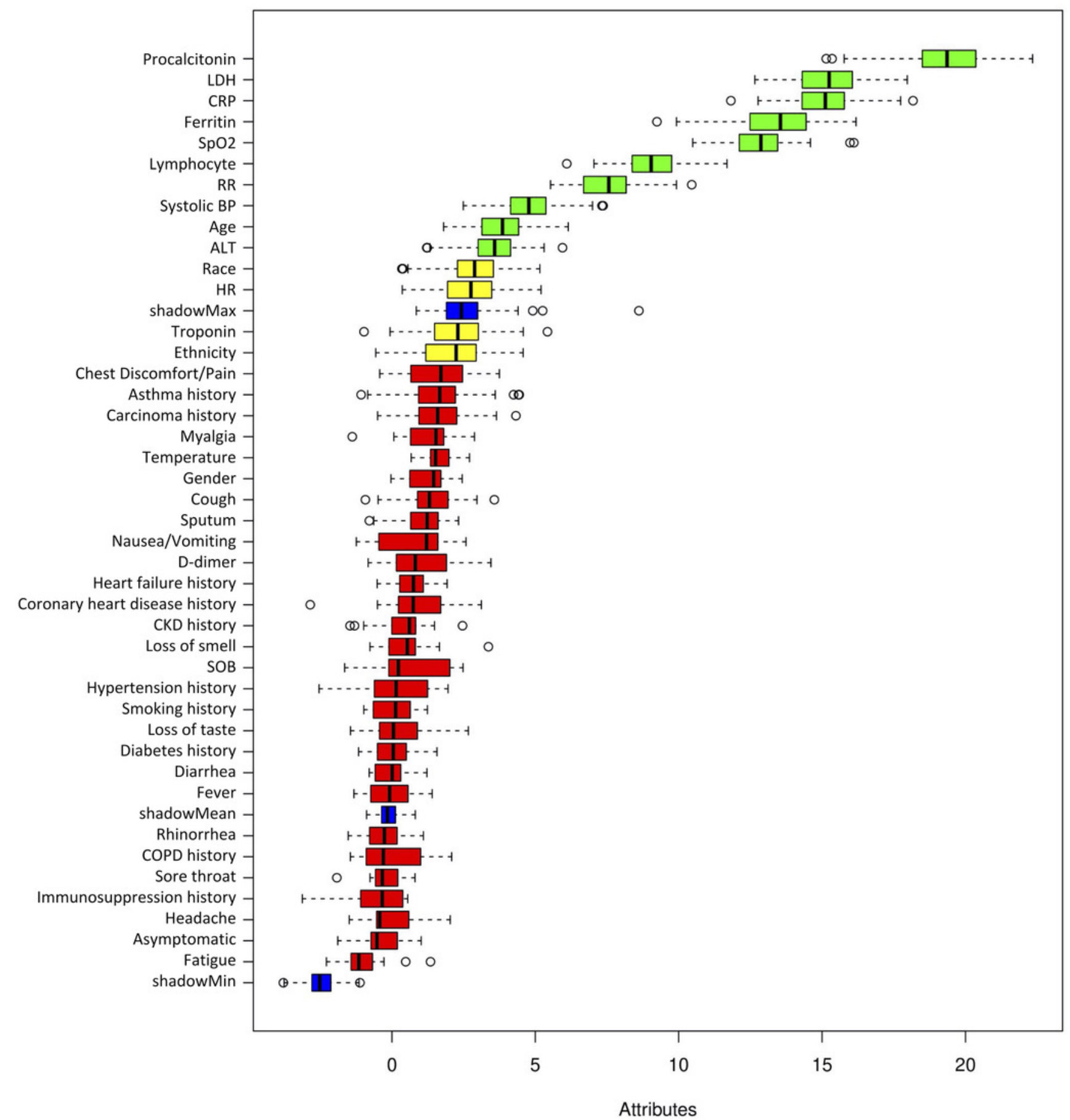


Figure 3

ROC and confusion matrix for prediction of ICU admission

(A) ROC and (B) confusion matrix for prediction of ICU admission of the DNN model.

(A)

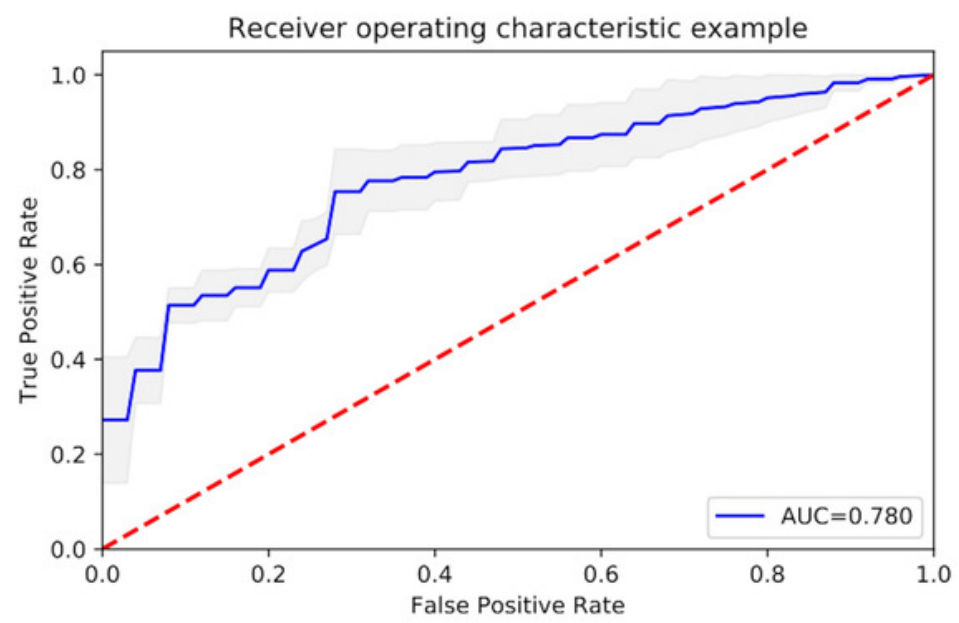

(B)

\begin{tabular}{c|c|c|}
\multicolumn{1}{c}{} & \multicolumn{1}{c}{$\begin{array}{c}\text { Predicted } \\
\text { ICU }\end{array}$} & $\begin{array}{c}\text { Predicted } \\
\text { non-ICU }\end{array}$ \\
\cline { 2 - 3 } Actual ICU & 19 & 6 \\
\hline \multirow{4}{*}{ Actual non-ICU } & & \\
\cline { 2 - 3 } & & \\
& & \\
& & \\
& & \\
\end{tabular}




\section{Figure 4}

Risk score stratification for ICU admission

Risk score stratification for ICU admission. Scores ranged from 0 to 5 , with 0 indicating the lowest risk and 5 being the highest risk of mortality. The numbers in the bar indicate the number of patients in the ICU (red) and non-ICU (blue) that were correctly predicted in the testing dataset.

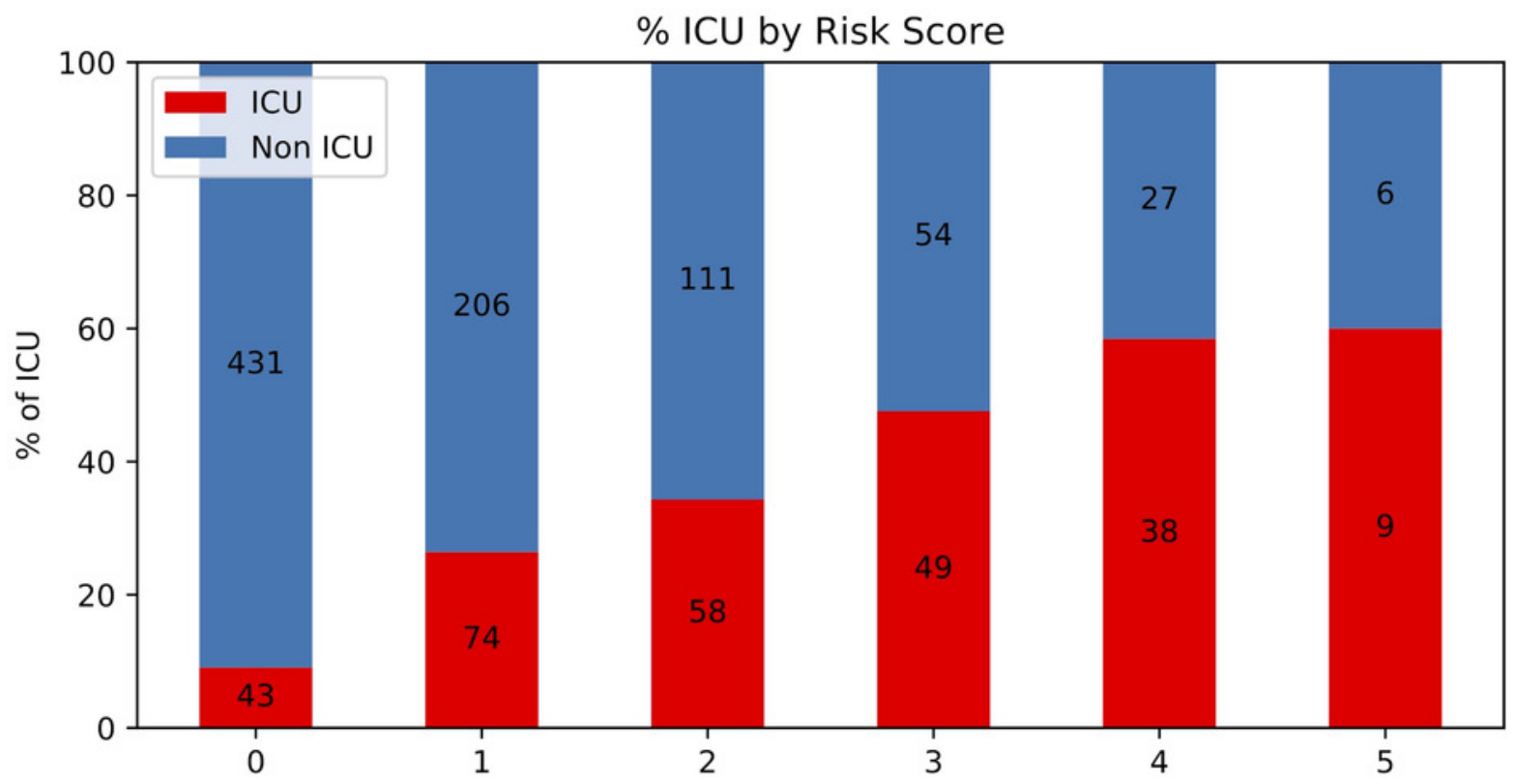




\section{Figure 5}

Ranking of clinical variables for predicting mortality.

Ranking of clinical variables for predicting mortality by Boruta algorithm. The $x$-axis is attribute of level of importance, where a larger number indicates relatively higher importance. The $y$-axis are laboratory test variables. The top statistically significant predictors were: age, LDH, procalcitonin, troponin, $\mathrm{CRP}, \mathrm{SpO} 2$, history of heart failure, respiratory rate, lymphocytes, ferritin, history of COPD, D-dimer, ALT, history of coronary heart disease, and systolic blood pressure. The top 15 variables were significant. 


\section{Mortality}

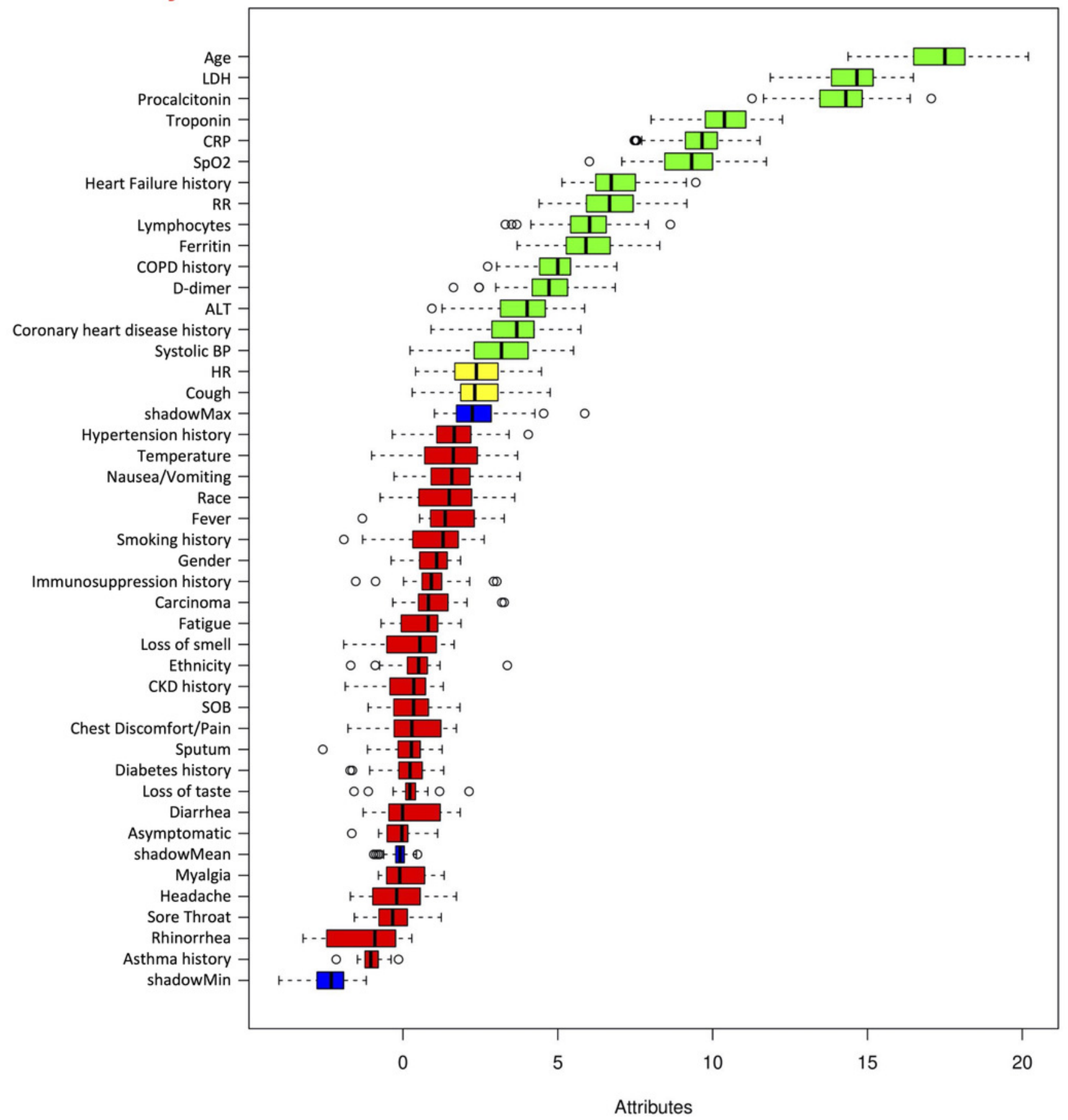


Figure 6

ROC and confusion matrix for prediction of mortality

(A) ROC and (B) confusion matrix for prediction of mortality of the DNN model.

(A)

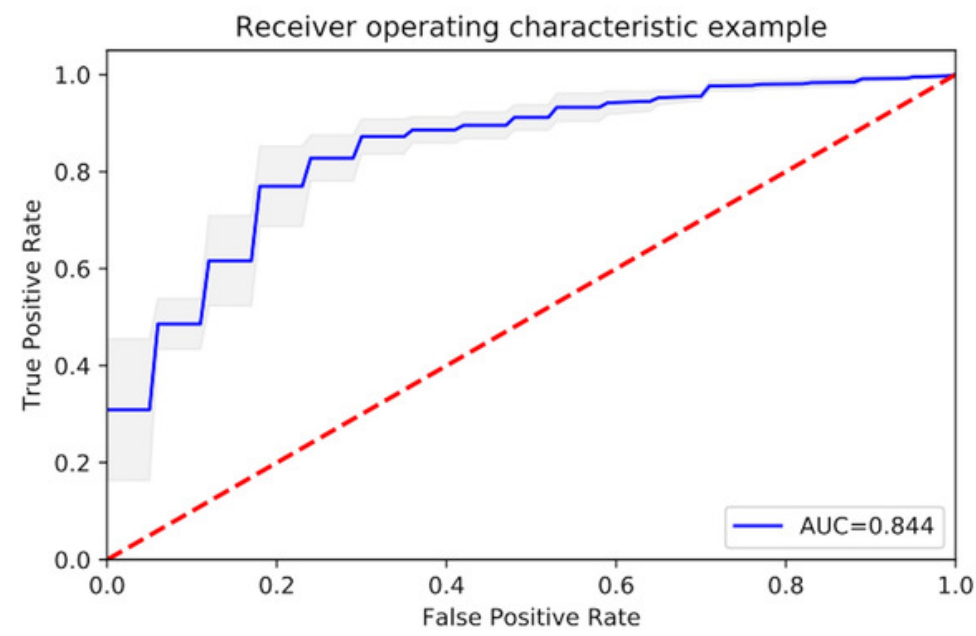

(B)

\begin{tabular}{c|c|c|}
\multicolumn{1}{c}{} & \multicolumn{1}{c}{$\begin{array}{c}\text { Predicted } \\
\text { death }\end{array}$} & $\begin{array}{c}\text { Predicted } \\
\text { survival }\end{array}$ \\
\cline { 2 - 3 } & & \\
\multirow{4}{*}{$\begin{array}{c}\text { Actual } \\
\text { death }\end{array}$} & 12 & 4 \\
\cline { 2 - 3 } $\begin{array}{c}\text { Actual } \\
\text { survival }\end{array}$ & 11 & 75 \\
\cline { 2 - 3 } & & \\
\cline { 2 - 3 } & & \\
& &
\end{tabular}




\section{Figure 7}

Risk score stratification for mortality

Risk score stratification for mortality. Scores ranged from 0 to 6 , with 0 indicating the lowest risk and 6 being the highest risk of mortality. The numbers in the bar indicate the number of patients in the ICU (red) and non-ICU (blue) that were correctly predicted in the testing dataset.

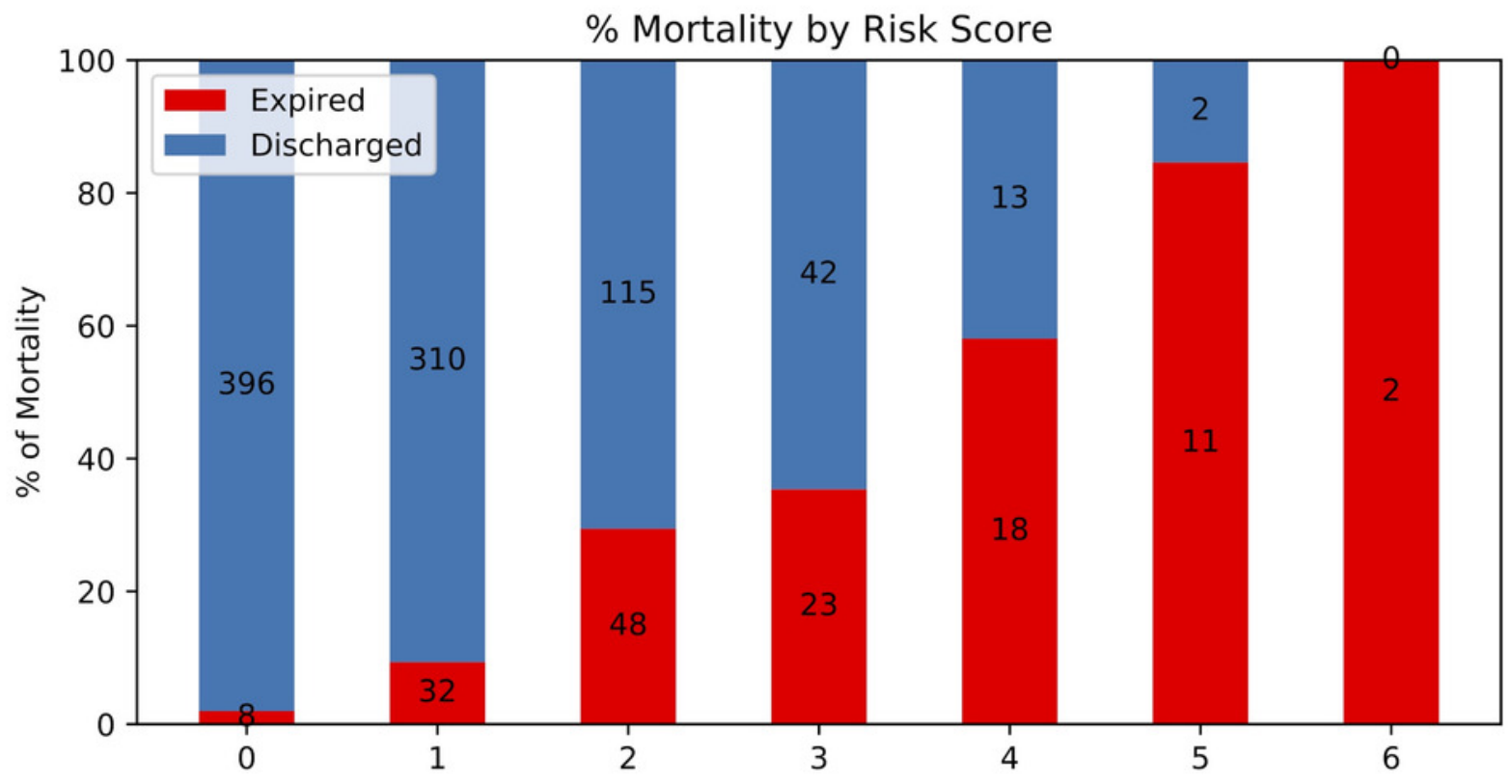




\section{Table $\mathbf{1}$ (on next page)}

Demographic characteristics, comorbidities, symptoms, imaging findings, vital signs, and laboratory findings of ICU versus non-ICU patients.

Demographic characteristics, comorbidities, symptoms, imaging findings, vital signs, and laboratory findings of ICU versus non-ICU patients. Group comparison of categorical variables in frequencies and percentages used $c^{2}$ test or Fisher exact tests. Group comparison of continuous variables in medians and interquartile ranges (IQR) used the Mann-Whitney $\mathrm{U}$ test. 
1 Table 1. Demographic characteristics, comorbidities, symptoms, imaging findings, vital signs, and

2 laboratory findings of ICU versus non-ICU patients. Group comparison of categorical variables in

3 frequencies and percentages used $\chi^{2}$ test or Fisher exact tests. Group comparison of continuous variables

4 in medians and interquartile ranges (IQR) used the Mann-Whitney U test.

\begin{tabular}{|c|c|c|c|}
\hline & \multicolumn{3}{|c|}{ Patients, No. (\%) } \\
\hline & $\begin{array}{c}\mathrm{ICU} \\
(\mathrm{n}=271)\end{array}$ & $\begin{array}{c}\text { Non-ICU } \\
(\mathrm{n}=837)\end{array}$ & $\mathrm{p}$ value \\
\hline \multicolumn{4}{|l|}{ Demographics } \\
\hline Age, median (range), y & $59(49,71)$ & $62(50,76)$ & 0.027 \\
\hline Sex & & & $<0.001$ \\
\hline Male & $183(67.5 \%)$ & $452(54.0 \%)$ & \\
\hline Female & $88(32.5 \%)$ & $385(46.0 \%)$ & \\
\hline Ethnicity & & & 0.153 \\
\hline Hispanic/Latino & $78(28.8 \%)$ & $223(26.6 \%)$ & \\
\hline Non-Hispanic/Latino & $148(54.6 \%)$ & $507(60.6 \%)$ & \\
\hline Unknown & $45(16.6 \%)$ & $107(12.8 \%)$ & \\
\hline Race & & & 0.003 \\
\hline Caucasian & $123(45.4 \%)$ & $453(54.1 \%)$ & \\
\hline African American & $13(4.8 \%)$ & $61(7.3 \%)$ & \\
\hline Asian & $20(7.4 \%)$ & $26(3.1 \%)$ & \\
\hline American Indian/Alaska Native & $2(0.7 \%)$ & $2(0.2 \%)$ & \\
\hline $\begin{array}{l}\text { Native Hawaiian or other Pacific } \\
\text { Islander }\end{array}$ & 0 & $1(0.1 \%)$ & \\
\hline More than One Race & 0 & $5(0.6 \%)$ & \\
\hline Unknown/Not Reported & $113(41.7 \%)$ & $289(34.5 \%)$ & \\
\hline \multicolumn{4}{|l|}{ Comorbidities } \\
\hline Smoking history & $61(22.6 \%)$ & $214(25.6 \%)$ & 0.332 \\
\hline Diabetes & $80(29.5 \%)$ & $220(26.3 \%)$ & 0.308 \\
\hline Hypertension & $126(46.5 \%)$ & $412(49.3 \%)$ & 0.442 \\
\hline Asthma & $23(8.5 \%)$ & $43(5.1 \%)$ & 0.054 \\
\hline COPD & $39(14.4 \%)$ & $126(15.1 \%)$ & 0.845 \\
\hline Coronary artery disease & $17(6.3 \%)$ & $76(9.1 \%)$ & 0.166 \\
\hline Heart failure & $18(6.6 \%)$ & $62(7.4 \%)$ & 0.787 \\
\hline Cancer & $15(5.5 \%)$ & $88(10.5 \%)$ & 0.016 \\
\hline Immunosuppression & $20(7.4 \%)$ & $64(7.7 \%)$ & 1.000 \\
\hline Chronic kidney disease & $20(7.4 \%)$ & $81(9.7 \%)$ & 0.276 \\
\hline \multicolumn{4}{|l|}{ Symptoms } \\
\hline Fever & $191(70.5 \%)$ & $547(65.4 \%)$ & 0.138 \\
\hline Cough & $191(70.5 \%)$ & $564(67.4 \%)$ & 0.368 \\
\hline Shortness of breath & $210(77.5 \%)$ & $557(66.5 \%)$ & 0.001 \\
\hline Fatigue & $56(20.7 \%)$ & $201(24.0 \%)$ & 0.282 \\
\hline Sputum & $25(9.2 \%)$ & $50(6.0 \%)$ & 0.071 \\
\hline Myalgia & $61(22.5 \%)$ & $192(22.9 \%)$ & 0.934 \\
\hline Diarrhea & $60(22.1 \%)$ & $201(24.0 \%)$ & 0.565 \\
\hline Nausea or vomiting & $48(17.7 \%)$ & $176(21.0 \%)$ & 0.258 \\
\hline
\end{tabular}




\begin{tabular}{|c|c|c|c|}
\hline Sore throat & $21(7.7 \%)$ & $61(7.3 \%)$ & 0.790 \\
\hline Rhinorrhea & $14(5.2 \%)$ & $36(4.3 \%)$ & 0.613 \\
\hline Loss of smell & $11(4.1 \%)$ & $34(4.1 \%)$ & 1.000 \\
\hline Loss of taste & $12(4.4 \%)$ & $42(5.0 \%)$ & 0.871 \\
\hline Headache & $80(9.6 \%)$ & $28(10.3 \%)$ & 0.724 \\
\hline Chest discomfort or chest pain & $43(15.9 \%)$ & $133(15.9 \%)$ & 1.000 \\
\hline \multicolumn{4}{|l|}{ Imaging studies } \\
\hline Abnormal chest $\mathrm{x}$-ray results & $227(92.1 \%)$ & $694(83.6 \%)$ & $<0.001$ \\
\hline Chest $\mathrm{x}$-ray findings & & & $<0.001$ \\
\hline Unilateral & $26(10.7 \%)$ & $140(20.7 \%)$ & \\
\hline Bilateral & $218(89.3 \%)$ & $536(79.3 \%)$ & \\
\hline \multicolumn{4}{|l|}{ Vital signs, median (IQR) } \\
\hline Heart Rate, bpm & $100(87,115)$ & $98(83,110)$ & 0.003 \\
\hline Respiratory rate, rate/min & $23(18,30)$ & $20(18,24)$ & $<0.001$ \\
\hline $\mathrm{SpO}_{2} \%$ & $93(87,96)$ & $94(92,97)$ & $<0.001$ \\
\hline Systolic blood pressure, $\mathrm{mmHg}$ & $122(108,137)$ & $127(114,144)$ & 0.003 \\
\hline Temperature, ${ }^{\circ} \mathrm{C}$ & $37.4(36.9,38.3)$ & $37.3(36.9,38.0)$ & 0.021 \\
\hline \multicolumn{4}{|c|}{ Laboratory findings at admission, median (IQR) } \\
\hline Alanine aminotransferase, $\mathrm{U} / \mathrm{L}$ & $37(22,59)$ & $29(17,51)$ & $<0.001$ \\
\hline Brain natriuretic peptide, $\mathrm{pg} / \mathrm{mL}$ & $276(81,1123)$ & $212(53,1143)$ & 0.177 \\
\hline C-reactive protein, $\mathrm{mg} / \mathrm{dL}$ & $12.8(6.9,22.1)$ & $7.2(3.2,13.3)$ & $<0.001$ \\
\hline D-dimer, $\mathrm{ng} / \mathrm{mL}$ & $401(257,831)$ & $353(217,657)$ & 0.012 \\
\hline Ferritin, ng/mL & $1132(582,1867)$ & $613(289,1234)$ & $<0.001$ \\
\hline Lactate dehydrogenase, U/L & $436(332,593)$ & $332(257,433)$ & $<0.001$ \\
\hline WBC, $\mathrm{x} 10^{3} / \mathrm{ml}$ & $8.1(6.1,11.6)$ & $7.3(5.5,9.4)$ & 0.001 \\
\hline Lymphocytes\% & $10.6(6.1,15.4)$ & $13.1(8.4,19.5)$ & $<0.001$ \\
\hline Procalcitonin, $\mathrm{ng} / \mathrm{mL}$ & $0.29(0.16,0.77)$ & $0.15(0.09,0.28)$ & $<0.001$ \\
\hline Troponin, $\mathrm{ng} / \mathrm{mL}$ & $0.01(0.01,0.01)$ & $0.01(0.01,0.01)$ & 0.596 \\
\hline
\end{tabular}

5 Abbreviation: COPD, chronic obstructive pulmonary disease. $\mathrm{IQR}$, interquartile range. $\mathrm{SpO}_{2}$, oxygen

6 saturation.

7

8 SI conversion factors: To convert alanine aminotransferase and lactate dehydrogenase to microkatal per

9 liter, multiply by 0.0167 ; C-reactive protein to milligram per liter, multiply by 10 ; D-dimer to nanomole 10 per liter, multiply by 0.0054 ; leukocytes to $\times 10^{9}$ per liter, multiply by 0.001 . 


\section{Table 2 (on next page)}

Performance indices for predicting ICU admission of the testing dataset

Performance indices for predicting ICU admission of the testing dataset. Abbreviations: area under the curve (AUC), accuracy, sensitivity, specificity, precision, recall, negative predictive value (NPV), positive predictive value (PPV) and F1 score (a harmonic mean of precision and recall). 
1 Table 2. Performance indices for predicting ICU admission of the testing dataset. Abbreviations: area 2 under the curve (AUC), accuracy, sensitivity, specificity, precision, recall, negative predictive value

3 (NPV), positive predictive value (PPV) and F1 score (a harmonic mean of precision and recall).

\begin{tabular}{|l|l|l|l|l|l|l|l|l|}
\hline & AUC & Accuracy & Sensitivity & Specificity & Precision & NPV & PPV & F1 \\
\hline Training & 0.751 & 0.703 & 0.707 & 0.701 & 0.437 & 0.879 & 0.437 & 0.540 \\
\hline Testing & 0.728 & 0.721 & 0.760 & 0.709 & 0.432 & 0.910 & 0.432 & 0.551 \\
\hline
\end{tabular}

4

5

6

7

8 


\section{Table 3 (on next page)}

Demographic characteristics, comorbidities, symptoms, imaging findings, vital signs, and laboratory findings of death versus non-death (discharged).

Demographic characteristics, comorbidities, symptoms, imaging findings, vital signs, and laboratory findings of death versus non-death (discharged). Group comparison of categorical variables in frequencies and percentages used $c^{2}$ or Fisher exact tests. Group comparison of continuous variables in medians and interquartile ranges (IQR) used the Mann-Whitney $U$ test. 
1 Table 3. Demographic characteristics, comorbidities, symptoms, imaging findings, vital signs, and

2 laboratory findings of death versus non-death (discharged). Group comparison of categorical variables in

3 frequencies and percentages used $\chi^{2}$ or Fisher exact tests. Group comparison of continuous variables in

4 medians and interquartile ranges (IQR) used the Mann-Whitney U test.

\begin{tabular}{|c|c|c|c|}
\hline & \multicolumn{3}{|c|}{ Patients, No. (\%) } \\
\hline & $\begin{array}{c}\text { Death } \\
(\mathrm{n}=142)\end{array}$ & $\begin{array}{l}\text { Non-death } \\
(\mathrm{n}=880)\end{array}$ & $\mathrm{p}$ value \\
\hline \multicolumn{4}{|l|}{ Demographics } \\
\hline Age, median (range), y & $76(66,84)$ & $59(49,72)$ & $<0.001$ \\
\hline Sex & & & 0.022 \\
\hline Male & $93(65.5 \%)$ & $484(55.0 \%)$ & \\
\hline Female & $49(34.5 \%)$ & $396(45.0 \%)$ & \\
\hline Ethnicity & & & 0.001 \\
\hline Hispanic/Latino & $23(16.2 \%)$ & $251(28.5 \%)$ & \\
\hline Non-Hispanic/Latino & $105(73.9 \%)$ & $504(57.3 \%)$ & \\
\hline Unknown & $14(9.9 \%)$ & $125(14.2 \%)$ & \\
\hline Race & & & 0.023 \\
\hline Caucasian & $91(64.1 \%)$ & $450(51.1 \%)$ & \\
\hline African American & $6(4.2 \%)$ & $61(6.9 \%)$ & \\
\hline Asian & $9(6.3 \%)$ & $33(3.8 \%)$ & \\
\hline American Indian/Alaska Native & $1(0.7 \%)$ & $2(0.2 \%)$ & \\
\hline $\begin{array}{l}\text { Native Hawaiian or other Pacific } \\
\text { Islander }\end{array}$ & 0 & $1(0.1 \%)$ & \\
\hline More than One Race & 0 & $5(0.6 \%)$ & \\
\hline Unknown/Not Reported & $35(24.6 \%)$ & $328(37.3 \%)$ & \\
\hline \multicolumn{4}{|l|}{ Comorbidities } \\
\hline Smoking history & $52(36.6 \%)$ & $204(23.2 \%)$ & 0.001 \\
\hline Diabetes & $48(33.8 \%)$ & $229(26.1 \%)$ & 0.067 \\
\hline Hypertension & $92(64.8 \%)$ & $402(45.8 \%)$ & $<0.001$ \\
\hline Asthma & $6(4.2 \%)$ & $51(5.8 \%)$ & 0.557 \\
\hline COPD & $23(16.2 \%)$ & $66(7.5 \%)$ & 0.002 \\
\hline Coronary artery disease & $39(27.5 \%)$ & $115(13.1 \%)$ & $<0.001$ \\
\hline Heart failure & $29(20.4 \%)$ & $47(5.4 \%)$ & $<0.001$ \\
\hline Cancer & $19(13.4 \%)$ & $78(8.9 \%)$ & 0.092 \\
\hline Immunosuppression & $8(5.6 \%)$ & $65(7.4 \%)$ & 0.598 \\
\hline Chronic kidney disease & $20(14.1 \%)$ & $75(8.5 \%)$ & 0.043 \\
\hline \multicolumn{4}{|l|}{ Symptoms } \\
\hline Fever & $81(57.0 \%)$ & $599(68.1 \%)$ & 0.012 \\
\hline Cough & $73(51.4 \%)$ & $628(71.4 \%)$ & $<0.001$ \\
\hline Shortness of breath & $102(71.8 \%)$ & $594(67.5 \%)$ & 0.333 \\
\hline Fatigue & $19(13.4 \%)$ & $216(24.5 \%)$ & 0.003 \\
\hline Sputum & $10(7.0 \%)$ & $58(6.6 \%)$ & 0.856 \\
\hline Myalgia & $15(10.6 \%)$ & $220(25.0 \%)$ & $<0.001$ \\
\hline Diarrhea & $27(19.0 \%)$ & $211(24.0 \%)$ & 0.239 \\
\hline Nausea or vomiting & $10(7.0 \%)$ & $192(21.8 \%)$ & $<0.001$ \\
\hline
\end{tabular}




\begin{tabular}{|c|c|c|c|}
\hline Sore throat & $7(4.9 \%)$ & $69(7.8 \%)$ & 0.300 \\
\hline Rhinorrhea & $4(2.8 \%)$ & $41(4.7 \%)$ & 0.386 \\
\hline Loss of smell & $2(1.4 \%)$ & $38(4.3 \%)$ & 0.106 \\
\hline Loss of taste & $2(1.4 \%)$ & $48(5.5 \%)$ & 0.035 \\
\hline Headache & $7(4.9 \%)$ & $90(10.2 \%)$ & 0.045 \\
\hline Chest discomfort or chest pain & $10(7.0 \%)$ & $151(17.2 \%)$ & 0.001 \\
\hline \multicolumn{4}{|l|}{ Imaging studies } \\
\hline Abnormal chest $\mathrm{x}$-ray results & $123(87.2 \%)$ & $720(84.6 \%)$ & 0.524 \\
\hline Chest x-ray findings & & & 0.214 \\
\hline Unilateral & $18(14.6 \%)$ & $142(19.7 \%)$ & \\
\hline Bilateral & $105(85.4 \%)$ & $577(80.3 \%)$ & \\
\hline \multicolumn{4}{|l|}{ Vital signs, median (IQR) } \\
\hline Heart Rate, bpm & $96(81,115)$ & $99(85,110)$ & 0.496 \\
\hline Respiratory rate, rate/min & $24(20,32)$ & $20(18,24)$ & $<0.001$ \\
\hline $\mathrm{SpO}_{2} \%$ & $93(88,96)$ & $94(92,96)$ & $<0.001$ \\
\hline Systolic blood pressure, $\mathrm{mmHg}$ & $127(105,142)$ & $125(113,143)$ & 0.568 \\
\hline Temperature, ${ }^{\circ} \mathrm{C}$ & $37.1(36.7,37.6)$ & $37.3(36.9,38.1)$ & $<0.001$ \\
\hline \multicolumn{4}{|c|}{ Laboratory findings at admission, median (IQR) } \\
\hline Alanine aminotransferase, U/L & $30.0(17.0,54.0)$ & $30.0(18.0,52.0)$ & 0.666 \\
\hline Brain natriuretic peptide, $\mathrm{pg} / \mathrm{mL}$ & $1652(452,4556)$ & $164(47,772)$ & $<0.001$ \\
\hline C-reactive protein, $\mathrm{mg} / \mathrm{dL}$ & $13.4(6.9,21.8)$ & $7.5(3.2,13.4)$ & $<0.001$ \\
\hline D-dimer, $\mathrm{ng} / \mathrm{mL}$ & $635(365,1753)$ & $333(213,606)$ & $<0.001$ \\
\hline Ferritin, ng/mL & $981(442,1657)$ & $640(308,1333)$ & $<0.001$ \\
\hline Lactate dehydrogenase, $\mathrm{U} / \mathrm{L}$ & $436(330,638)$ & $333(257,434)$ & $<0.001$ \\
\hline WBC, $x 10^{3} / \mathrm{ml}$ & $8.7(6.4,12.3)$ & $7.3(5.5,9.5)$ & 0.001 \\
\hline Lymphocytes $\%$ & $8.9(5.3,13.6)$ & $13.3(8.7,19.4)$ & $<0.001$ \\
\hline Procalcitonin, ng/mL & $0.34(0.18,1.26)$ & $0.15(0.090,0.28)$ & $<0.001$ \\
\hline Troponin, ng/mL & $0.02(0.01,0.07)$ & $0.01(0.01,0.01)$ & $<0.001$ \\
\hline
\end{tabular}

5 Abbreviation: COPD, chronic obstructive pulmonary disease. IQR, interquartile range. $\mathrm{SpO}_{2}$, oxygen

6 saturation.

7

8 SI conversion factors: To convert alanine aminotransferase and lactate dehydrogenase to microkatal per

9 liter, multiply by 0.0167 ; C-reactive protein to milligram per liter, multiply by 10 ; D-dimer to nanomole 10 per liter, multiply by 0.0054 ; leukocytes to $\times 10^{9}$ per liter, multiply by 0.001 . 


\section{Table 4(on next page)}

Performance indices for predicting mortality

Performance indices for predicting mortality of the testing dataset. Abbreviations: area under the curve (AUC), accuracy, sensitivity, specificity, precision, recall, negative predictive value (NPV), positive predictive value (PPV) and F1 score (a harmonic mean of precision and recall). 
1 Table 4. Performance indices for predicting mortality of the testing dataset. Abbreviations: area under the 2 curve (AUC), accuracy, sensitivity, specificity, precision, recall, negative predictive value (NPV),

3 positive predictive value (PPV) and F1 score (a harmonic mean of precision and recall).

\begin{tabular}{|l|l|l|l|l|l|l|l|l|}
\hline & AUC & Accuracy & Sensitivity & Specificity & Precision & NPV & PPV & F1 \\
\hline Training & 0.852 & 0.892 & 0.706 & 0.922 & 0.589 & 0.952 & 0.589 & 0.642 \\
\hline Testing & 0.844 & 0.853 & 0.750 & 0.872 & 0.522 & 0.949 & 0.522 & 0.616 \\
\hline
\end{tabular}

4

5

6

7 